Vanessa Martínez Ardila*, Ángela Liliana Dotor Robayo**

\title{
El diseño y experimentación textil como representación y resignificación de la mujer en el postconflicto en Colombia ${ }^{1}$
}

The textile design experimentation as representation and
resignification of women in the post-conflict in Colombia

Cómo citar:

Martínez, V. \& Dotor, A. (2019). El diseño y experimentación textil como representación y resignificación de la mujer en el postconflicto en Colombia. Designia, 7(2), 67-87.

${ }^{1}$ Universidad de Bogotá Jorge Tadeo Lozano, reflexión sobre proyecto de grado finalizado.

* Vanessa Martínez Ardila graduada de la Universidad Jorge Tadeo Lozano en Diseño y Gestión de la Moda. Hizo parte del primer semillero que inauguró su carrera llamado Origen-1 a cargo de Carolina Obregón. Sus áreas de interés son el vestuario, el styling, la lencería y el trabajo social. Actualmente desarrolla su marca personal

y hace parte de El Olimpo, un colectivo que desarrolla diferentes proyectos comunitarios y de artivismo en el barrio Santa fé en Bogotá.

E-mail: vanessa.martinez@utadeo.edu.co ORCID: https://orcid.org/0000-0001-6721-3471
Palabras clave:

Diseño social, reinserción, cocreación.

Key words:

Social design, reinsertion, co-creation.

Recibido: 25/05/2019

Aceptado: 28/08/2019

**PhD(c) Diseño, Universidad de Palermo,Buenos Aires. Maestra en Dirección de Marketing. Profesional en Diseño de modas y Textiles. Profesora Asociada Universidad de Bogotá Jorge Tadeo Lozano en la Escuela de Diseño, Fotografía y realización audiovisual. Profesora de taller de diseño con especial interés en proyectos que piensan el diseño para la sostenibilidad y los estudios culturales de la moda.

E-mail: angelal.dotorr@utadeo.edu.co ORCID: http://orcid.org/0000-0002-4296-7848 


\section{Resumen:}

Tras la firma de los Acuerdos de Paz, la resocialización de excombatientes y víctimas de la guerra es uno de los principales retos del postconflicto en Colombia, y en él se abordan diferentes componentes que comprenden una reincorporación integral en la sociedad.

En este contexto, este artículo se presenta como reflexión sobre el proyecto de investigación-creación en diseño llamado "Mi historia aún no termina", planteado como una plataforma de reincorporación social y resignificación de la mujer a través de talleres y laboratorios vivos de diseño con reflexión sobre textil, bordado y tejido, bajo principios de colaboración y cocreación.

El proyecto fue implementado en 2018 en Bogotá-Colombia como una perspectiva de diseño con enfoque social, que se desarrolló a través de un taller de creación que partió de la conceptualización testimonial de la violencia, el cuerpo, la verdad y su valor simbólico para la resignificación de los sujetos, lo que se tradujo en talleres de diseño para la higiene social y la construcción de tejido social. Como resultado de la experiencia se obtuvo una colección de indumentaria (como representación de las mujeres participantes), una exposición de la exploración arte-tejido, y un grupo de mujeres empoderadas en su tejido social.

\section{Abstract:}

After the signing of the Peace Agreements, the re-socialization of ex-combatants and victims of the war is one of the main challenges of the post-conflict in Colombia, where different components include an integral reincorporation into society are addressed.

In this context, this article is presented as a reflection on the research-creation project in design called "My story has not ended yet", proposed as a platform for social re-integration and re-signification of women through workshops and living design and reflection laboratories on textiles, embroidery and weaving under the principles of collaboration and co-creation. 
It was developed in 2018 in Bogotá, Colombia, as a design perspective with a social approach through a creation workshop based on the testimonial conceptualization of violence, the body, truth and its symbolic value to re-signify the subjects, which translated into design workshops for social hygiene and into the construction of social fabrics. From this experience a collection of clothing was obtained as a representation of women, as well as an exhibition of art-tissue exploration and a group of women empowered in their social fabric.

\section{INTRODUCCION}

Después de la firma de los Acuerdos de Paz entre el Gobierno colombiano y la guerrilla de las FARC (Fuerzas Armadas Revolucionarias de Colombia) en 2016, Colombia se ha dedicado a aprender a construir la paz en diferentes campos; entre ellos, el diseño de modas y textil. Uno de los objetivos es la reincorporación de excombatientes y víctimas, que a su vez es uno de los principales retos del postconflicto, ya que aborda diferentes componentes sociales, culturales y económicos.

En la búsqueda de una reincorporación integral en la sociedad, todos los colombianos quieren la paz, pero ¿qué hacer en el posconflicto cuando las instituciones públicas son insuficientes para enfrentar la reintegración de manera aislada y cuando las organizaciones sin ánimo de lucro no dan abasto con las demandas sociales?

Es en este marco donde surge la pregunta: ¿Cuál es el aporte que puede tener el diseño de modas y textil en el postconflicto? Se trata de una pregunta compartida por diferentes áreas interesadas en apoyar la reconstrucción del tejido social colombiano, reconstrucción a la que el diseño de modas no puede ser indiferente.

Para atender este llamado se propuso el proyecto de investigación-creación en diseño "Mi historia aún no termina" (MHANT), que se interesa en abordar la reintegración desde la perspectiva de género, en particular a través de la reflexión sobre el cuerpo y la sexualidad con naturalidad para la reconstrucción de la identidad personal, 
con la firme convicción de que es indispensable tratar temas tabú para construir la paz, tratar el problema de la violencia, el cuerpo y su libertad para construir la paz, y emplear metodologías de pensar el diseño y su saber hacer mediante el ejercicio codisciplinar y colaborativo en el diseño mismo.

Para este fin se planteó una metodología de diseño para la resocialización, la cual partió de utilizar los principios y prácticas del diseño como formas de conceptualización de la realidad contextual para representarla mediante productos de diseño textil y de indumentaria. De esa forma, la reflexión sobre el contexto social se trabajó bajo la perspectiva del psicoanálisis para la aprehensión inicial del problema, y para una posterior consideración se usó una aproximación autónoma y representación tranquila.

La experiencia de trabajar con laboratorios vivos, en este caso con mujeres sujetos del conflicto armado en Colombia, excombatientes de la guerrilla de las FARC, indígenas desplazadas por la violencia y mujeres campesinas y rurales víctimas del conflicto, permite entender el rol de la mujer, resignificarla y empoderarla socialmente mediante el proceso conceptual y de diseño. Se propone entonces al diseño como agente de transformación social, no para proponer asistencialismo, sino para sanar y, de paso, desarrollar formas y grupos sociales como tejido social y como tejido productivo.

El papel del diseñador como líder y transformador social en esta iniciativa implica que debe ser mediador y guía en el camino de exploración de la experiencia personal de cada uno de los participantes, pues no solo tiene que escuchar diferentes testimonios, sino que debe hacer posible que las historias personales se conceptualicen y se analicen, para así poder canalizar toda esa referencia sensorial en elementos figurativos, artísticos y textiles asociados al proceso de creación y de diseño de cada mujer; proceso en el que cada una de ellas plasma de manera simbólica su cuerpo y su historia para generar un efecto de higiene mental que desemboca en una gran expresión por medio del diseño.

Por lo anteriormente dicho, el proceso llevado acabo implicó, entre otras dinámicas, la realización de talleres que pretendían atacar el desconocimiento, la vergüenza y los prejuicios acerca de la sexualidad y la violencia sexual, que al no ser tratados actualmente de manera directa, generan diferentes formas de violencia que afectan a la sociedad en general, pero que se exacerban en el caso de escenarios y personas cercanas al conflicto; como en el caso de excombatientes y víctimas que resultan revictimizadas, por ejemplo, con la discriminación que no acepta las diferencias y, al contrario, culpa y avergüenza a las mujeres violentadas y fomenta las estructuras básicas de la violencia (Segato, 2014). 
Este artículo se presenta entonces como un ejemplo de reflexión sobre cómo el diseño puede ser parte de la resocialización, la recuperación y la reinserción de las personas que fueron parte del conflicto armado en Colombia. A continuación, se presenta un breve marco teórico y la metodología que se usó en los talleres y en el desarrollo del proyecto; al final, se presentan las conclusiones y los resultados del mismo.

\section{Trama histórica del conflicto en Colombia}

La siguiente semblanza debe verse como un paneo general de la historia del conflicto y la guerra en Colombia y sus víctimas, ya que históricamente el país ha vivido en guerra desde la Colonia; incluso después de la independencia se conocieron las guerras fratricidas en la Patria Boba.

Cuando llegaron los liberales al poder hacia 1820 se dio la abolición de la esclavitud y esto trajo una serie de conflictos de la posguerra libertaria; un siglo después vino la época de La Violencia, llamada así por la lucha de los seguidores entre partidos. Sumado a esta situación, hacia 1960 surgieron otros problemas sociales más profundos, como la pobreza, que llegó a estados de precariedad extremos. Y a raíz de tantas desigualdades, nació un conflicto por territorios y capitales, se dieron los movimientos guerrilleros, se agudizó el narcotráfico como pilar económico de las principales ciudades, trayendo como respuesta guerra al Estado, grupos paramilitares, sicariato y autodefensas (Atehortúa Cruz, A. L. \& Rojas Rivera, M. 2008).

No se puede entonces hablar de un solo tipo de violencia o de víctimas, pues la historia de Colombia está atravesada por momentos convulsionados que dejan en el camino víctimas de los diferentes tipos de violencia, y una marcada distribución de la vulnerabilidad en poblaciones rurales, campesinas y mujeres.

En estos diferentes marcos de guerra y conflicto se generó una estructura de orden y poder político patriarcal, en el que vale la pena aclarar que no son los hombres los enemigos de las mujeres sino el poder patriarcal el que feminiza y pone en marcha diferentes formas de violencia y crueldad hacia ellas (Segato, 2014). 
Bajo esta mirada, el conflicto armado en Colombia se ha desarrollado alrededor de la pobreza, el narcotráfico, el control de las tierras y la política de Estado, llevando consigo situaciones de precariedad, vulneración y violencia que conforman la historia del país, hasta el punto de una verdadera crónica de la violencia que evolucionó y se agudizó con la aparición de los carteles del narcotráfico, las autodefensas y las guerrillas. Esto generó más financiación de la guerra por parte del Estado, llevando a que la política de gobierno en los últimos 20 años fuera más armas de guerra. Este panorama es bien conocido a nivel internacional, pues trascendió nuestras fronteras narrando a diferentes países las crónicas de narcos, sicarios, bombas, guerra y guerrilla.

La lucha del Estado contra todos los grupos al margen de la ley fue uno de los puntos obligados dentro del plan de gobierno de cada uno de los presidentes de los últimos 70 años. Por el contrario, la apuesta por soluciones pacíficas y de diálogo fue insuficiente en contraste con las acciones de guerra y confrontación armada.

El Centro de Memoria Histórica del país ha reportado que desde 1970 hasta 2012 el conflicto armado en Colombia ha causado la muerte de 218.094 personas, donde el $19 \%$ de esta cifra pertenece a combatientes y el $81 \%$ a civiles (Estadísticas del conflicto armado en Colombia, 2012). El Registro Único de Víctimas (RUV) refleja que hasta la fecha, en 52 años de conflicto interno, hay 8.760 .290 víctimas de crímenes que comprenden asesinatos, desapariciones, violaciones, torturas o desplazamientos (Unidad para las Víctimas, 2018). Estas asombrosas cifras y datos, así como la laceración social que las siguieron, dieron origen a una negociación para la paz que posibilitara una historia diferente para el país.

El Centro de Memoria Histórica también revela que el conflicto armado en Colombia ha dejado 1.754 víctimas por violencia sexual desde 1985 hasta 2012 (Estadísticas del conflicto armado en Colombia, 2012). Y es esta la razón que ha impulsado al presente proyecto a trabajar temas desde el cuerpo, la sexualidad y el género, pues el desconocimiento de esta situación silencia a la víctima y permite que el victimario siga ejerciendo dominio sobre la misma. Es importante, sin embargo, tener en cuenta que no se trata de un hecho exclusivo del conflicto. Lamentablemente, Salud Pública de Colombia también ha arrojado cifras alarmantes: en el 73 \% de casos de abuso sexual en Colombia las víctimas son niñas (EL TIEMPO, 2016).

A continuación se presenta un listado de 16 tipos de hechos victimizantes (incluyendo una categoría indefinida). Al examinar el listado, se puede notar el aumento de los hechos y de las víctimas hasta antes del proceso de paz con la guerrilla, donde las cifras comienzan a disminuir. Según los datos de la Unidad para la Atención y Reparación Integral a las Víctimas (2019), la violencia ha afectado a casi 
la misma cantidad de hombres y mujeres: si bien la mayoría de las víctimas por la organización misma del combate son hombres, así como en las organizaciones del narcotráfico, hay que tener en cuenta que un amplio porcentaje de mujeres muere por cercanía territorial o algún tipo de relación con el conflicto, pero pocas veces por ser combatiente.

\begin{tabular}{|c|c|c|c|c|c|}
\hline & Hombre & Mujer & LGBTI & No Informa & Total general \\
\hline Abandono o Despojo de Tierras & 1654 & 1580 & 0 & 175 & 3409 \\
\hline Acto terrorista & 21194 & 144161 & 27 & 1665 & 37302 \\
\hline Amenaza & 65557 & 64382 & 531 & 3510 & 133980 \\
\hline Confinamiento & 370 & 354 & 2 & 114 & 840 \\
\hline $\begin{array}{l}\text { Delitos contra Ia integridad } \\
\text { sexual }\end{array}$ & 1741 & 14172 & 212 & 582 & 16707 \\
\hline Desaparición forzada & 29201 & 28239 & 18 & 2217 & 59675 \\
\hline Desplazamiento & 25322 & 254184 & 1956 & 25949 & 535309 \\
\hline Homicidio & 85069 & 86099 & 111 & 9665 & 180944 \\
\hline Lesiones Personales Fisicas & 3930 & 2287 & 28 & 166 & 6411 \\
\hline Lesiones Personales Psicologicas & 220 & 182 & 2 & 11 & 415 \\
\hline Minas Antipersonal & 5338 & 939 & 1 & 126 & 6404 \\
\hline Perdida de Muebles o Inmuebles & 17039 & 14582 & 29 & 1939 & 33589 \\
\hline Secuestro & 14748 & 5730 & 18 & 672 & 21168 \\
\hline Sin informacion & 281 & 197 & 0 & 25 & 503 \\
\hline Tortura & 3490 & 2771 & 28 & 174 & 6463 \\
\hline $\begin{array}{l}\text { Vinculacion de Niños y Ado- } \\
\text { lescentes }\end{array}$ & 2952 & 1793 & 6 & 131 & 4882 \\
\hline Total general & 506004 & 491907 & 2969 & 47121 & 1048001 \\
\hline
\end{tabular}

Tabla 1. Hechos victimizantes por género. Fuente: Fuente: Unidad de Víctimas. Elaboración propia.

En este contexto es claro que toda la sociedad colombiana tiene un compromiso con la reconstrucción de la sociedad desde diferentes perspectivas. Los diferentes hechos proponen un desafío diferente pero integral al que todas las áreas pueden contribuir. En el siguiente apartado se explicará cómo el diseño puede incidir en este contexto. 


\section{El sentido del diseño social y la representación de los sujetos}

Podríamos decir que cualquier disciplina es social, ya que pertenece y se desarrolla en un contexto social e intenta satisfacer necesidades tanto de la sociedad como de quien se expresa; en este caso en particular, a través del diseño, bajo la mirada amplia del diseño social como práctica contrapuesta al mercado caníbal, y provista de un fuerte discurso de entorno desarrollador de población, en el que se puede reflexionar sobre el efecto del diseño en un grupo social determinado (Ledesma, 2013).

\section{En palabras de la editorial del Glocal Design Magazine: \\ "El diseño es social por naturaleza. Es una disciplina integral que abarca dentro sus elementos principales al usuario, al individuo, su sociedad y la interacción con su entorno. Tener un acercamiento social consciente e informado en cualquier práctica de diseño contribuye al desarrollo del bienestar social, amplifica el impacto positivo en cualquier proyecto". (Glocal Design Magazine, 2018)}

El diseño social puede entenderse como un gran compilado o posibilidades de coexistencia y desarrollo social a partir del diseño, el cual permite entender el ejercicio de proyección como una plataforma en la que cohabitan la cognición, la conceptualización, la creatividad y el desarrollo de producto. En otras palabras, es un tránsito del contexto a la estética, que puede visualizarse como el tránsito del diseño de la trama cognitiva a la trama estética (Horta, 2016) y de representación. Una manera posible de lograr una representación es proponer el proyecto estético como un proyecto de identidad, identidad que, en palabras de Arendt, estaría provista del discurso personal y de las acciones (Terenzi, 2012), posibilitando la reconstrucción y resignificación de la identidad de cada uno de los sujetos participantes; para el caso, las mujeres.

Siguiendo esta línea, el taller de diseño se plantea como espacio de coexistencia. En este proyecto, se refiere a mujeres que hicieron parte del conflicto: guerrilleras, campesinas, indígenas, algunas combatientes, otras víctimas de violencia y desplazamiento, pero todas ellas con una carga de vulnerabilidad por el conflicto mismo. A través del proceso de diseño se desarrolló una estrategia para contribuir a su reparación y atención integral.

A pesar de que se puede pensar el diseño de modas y de indumentaria con este enfoque social, muchas percepciones, como la de Pierre Bourdieu, enmarcan la moda como un ejercicio frívolo que ha pretendido ciertas distinciones sociales; es decir, como un ejercicio de gusto y de necesidad de diferenciación simbólica que revela el 
verdadero poder adquisitivo mediante el lujo, convirtiendo a la moda en un gran juego de lucha por ese capital simbólico llamado distinción (Bourdieu, 2002).

Quizás esto es así porque a través de la historia la opulencia se ha convertido en la bandera de la moda: la "necesidad" de mostrar más y de proponer al mundo una apariencia. El presente estudio, en cambio, se plantea el diseño de producto textil y de moda bajo una perspectiva de desarrollo para la sociedad mediante un sistema de diseño de indumentaria que se acerca a la creación de bienestar social y que, por el contrario, trabaja en el cierre de brechas sociales, de discriminación y de violencia.

A diferencia de algunos de los productores de moda, que se han enfocado en producir estéticas solo para la construcción de la apariencia temporal, el lujo momentáneo y la generación de capital, el planteamiento de producción estética e indumentaria para la reinserción social propone dinámicas de producción colaborativa y solidaria, respetando las identidades de los individuos y las buenas prácticas sociales; esto es, el respeto y la inclusión del otro como sujeto.

Así, bajo una mirada sistémica y social, se plantea repensar la moda como un sistema socio-ético y socio-estético diferente al conocido, que sirve como espacio de liberación, de desahogo y de reflexión con conciencia; un espacio de reconstrucción social que forma personas creativas y productivas mediante la producción de indumentaria y textiles creativos y que es respetuoso con las personas y además crea un espacio de diálogo donde cada estética desarrollada cuenta una historia ligada a cada individuo que, al compartir sus experiencias, se conecta, se sana, comprende, encuentra explicaciones y reflexiona, en este caso, sobre su cuerpo, la sexualidad y el género.

Es claro que poniendo en marcha acciones sociales de diseño se logra lo que Niklas Luhmann (Urteaga, 2010) señalaba: "los sistemas vivos producen vida, los sociales producen conciencia". Se puede afirmar entonces que este proyecto generó una dinámica y metodología de creación de moda que no solo produjo diseño y estética, sino que además permitió crear un espacio de conciencia y liberación para la resignificación de la mujer. 
En resumen, el diseño tiene fuertes bases en el arte; y en su aspecto social, si bien al principio fue desarrollado como un rasgo diferenciador de clases, como algo superficial y voraz por su necesidad de innovación, en la contemporaneidad el diseño es un ejercicio social que permite resurgir, en un escenario donde el bienestar de los individuos y de las comunidades es el verdadero objetivo. No se trata únicamente de la búsqueda de mejoras en la producción y en la materia prima, sino que debe ser visto en su conjunto como un escenario de desarrollo de capacidades que mejora las condiciones de vida de cualquier grupo. En el proyecto "Mi historia aún no termina", se trata de ofrecer un escenario diferente de reintegración que, al tratar un tema del cual no se preocupan la mayoría de instituciones - por no ser considerado primordial- busca ser integral.

\section{Cuerpo, sexualidad y género como reflexión personal}

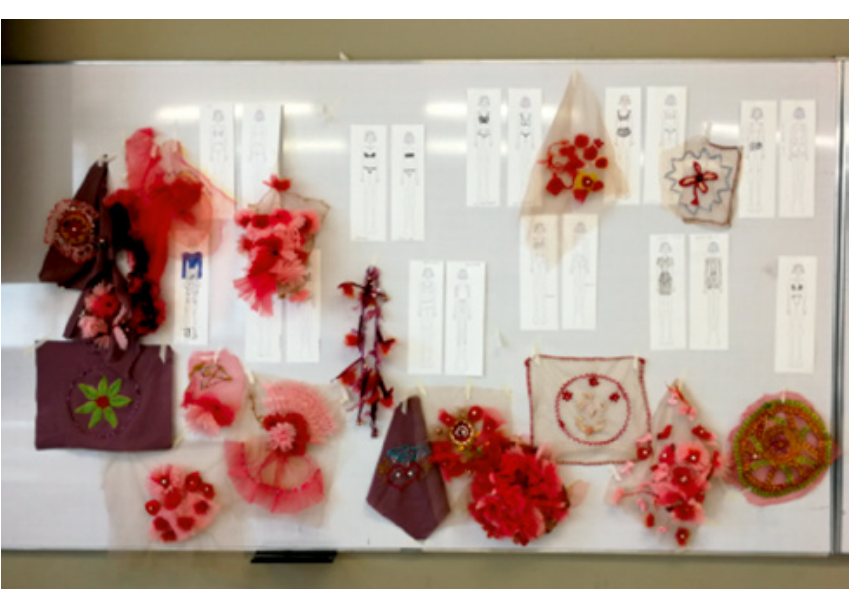

Figura 1. Cuerpo, historia y narrativa en textil.

Fuente: autoras.

Hablar de cuerpo, sexualidad y género es también hablar de autorreconocimiento, autodeterminación e introspección; para este fin, es necesario entender los diferentes conceptos para darle profundidad y espacio a la reconstrucción o resignificación del individuo, de su identidad y así dar paso a un proceso de reflexión del yo como mujer, o como sujeto e individuo. 
Como siguiente concepto se debe hablar del cuerpo, el cual, para este proyecto, se aleja de la dualidad cartesiana y se define como el ser; el individuo es visto como en la definición de la RAE: "Aquello que tiene extensión limitada, perceptible por los sentidos" (RAE, 2018). Dichos cuerpos son todos los sujetos e individuos donde, claramente, se define a los humanos; y en este sentido la definición del cuerpo es parte importante en la construcción de la identidad para la representación de cada sujeto del postconflicto.

Algunas miradas más básicas y conservadoras asumen el género como una característica del cuerpo según la categoría biológica más evidente: el sexo, el cual es una denominación según los genitales - macho y hembra - que a su vez son vistos arcaicamente como lo masculino o femenino. Pero el cuerpo no siempre coincide con la sexualidad ni con el género. Por eso es importante entender que las teorías sobre el tema han desarrollado imaginarios de identidad, para tener una mejor noción de lo que define a los individuos. Así, la identidad "es un constructo social que representa la noción que la persona tiene de sí misma" (Vargas-Trujillo, 2007); el cuerpo es, para el caso, la presencialidad, el ser y su corporalidad en uno solo.

Por otra parte, está el género entendido como una categoría que describe el conjunto de normas socioculturales que definen lo femenino, que incluso podría entenderse como mandato: el cómo deben pensar, sentir y actuar las personas según la apariencia de su cuerpo/sexo, así como la forma de pensar sobre la mujer, que hace que tenga un rol muy definido en los espacios de conflicto.

Finalmente, la sexualidad es la amalgama de las características y valoraciones que las personas utilizamos para describir nuestro sexo, género y orientación sexual (Vargas-Trujillo, 2007), lo que coincide con la visión de la filósofa Judith Butler, quien plantea que el género es una construcción social (Butler, 2007) y que plantea a las mujeres como sujetos femeninos, sujetos sexuales y fértiles. Con esto se va reduciendo a muchas mujeres del conflicto colombiano a sujetos biológicamente identificados como objetivos de guerra y de violencia. 
Teniendo claras las definiciones, podemos observar que en la literatura, la historia de la violencia y el conflicto, en general, se recogen los tratos más crueles que han sufrido las mujeres. Hechos que las identifican como sujetos víctimas de violencia de género, actos perversos y que postulan diferentes tipos de crueldad y, en lo específico y común, violencia sexual bajo diferentes formas como embarazo forzado, esclavitud sexual, tortura y obligación de aborto (Jiménez, 2016). Estas prácticas normalizadas en la esfera de la violencia hacen que el sujeto como mujer se disminuya y se desvalorice.

No obstante, no todo se reduce a este tipo de violencia. Tanto en el conflicto como fuera de él, la sociedad lucha con identidad de género, apartándose cada vez más del binarismo femenino-masculino, para abrir una serie de posibilidades nuevas tanto para hombres como mujeres. Estas discusiones siguen siendo un tabú en esferas tradicionales y patriarcales, pues no es fácil entender lo que siente otro o cómo se percibe "el otro" cuando ya se tiene una preconcepción de la identidad sexual como identidad de género.

En la agenda del postconflicto, desafortunadamente, la prioridad no es el desarrollo de proyectos que permitan espacios de discusión sobre la violencia de género, espacios que permitirían entender los cuerpos y con ellos la sexualidad y sus derechos de aparición e individualidad. En lo que se corrobora al revisar los planes y proyectos de inversión y la propia misión de los organismos creados para manejar la reparación de las víctimas estos temas simplemente no existen. Ello se debe a que los organismos públicos se concentran en los asuntos considerados prioritarios, tales como procedimientos administrativos, documentación de las bases de datos y la reubicación de las víctimas, (Unidad para la atención y reparación integral a las victimas, 2019).

Sin embargo, abordar esta problemática de la reconstrucción del individuo y de la reconstrucción de la identidad de género es una necesidad real en los diferentes escenarios, pues se entiende que desde estas características es desde donde se define la identidad que permite un desarrollo integral de la persona, hace sujetos más libres, más capaces y más dispuestos para enfrentar la vida. De esta forma, las personas también deben aceptar y entender todas las formas de género y su derecho a mostrarlo socialmente en igualdad de términos, contribuyendo con ello a combatir la violencia de género desde sus formas más básicas. 
"Al mismo tiempo, y ello no es contradictorio, cuando la identidad es un recurso, se crea una valorización de la autonomía y de la identidad, como valor, como subjetividad contra las identidades atribuidas, pesadas o impuestas las que frenan (...) la libertad del ciudadano".

(Dubet, 1989, p. 526).

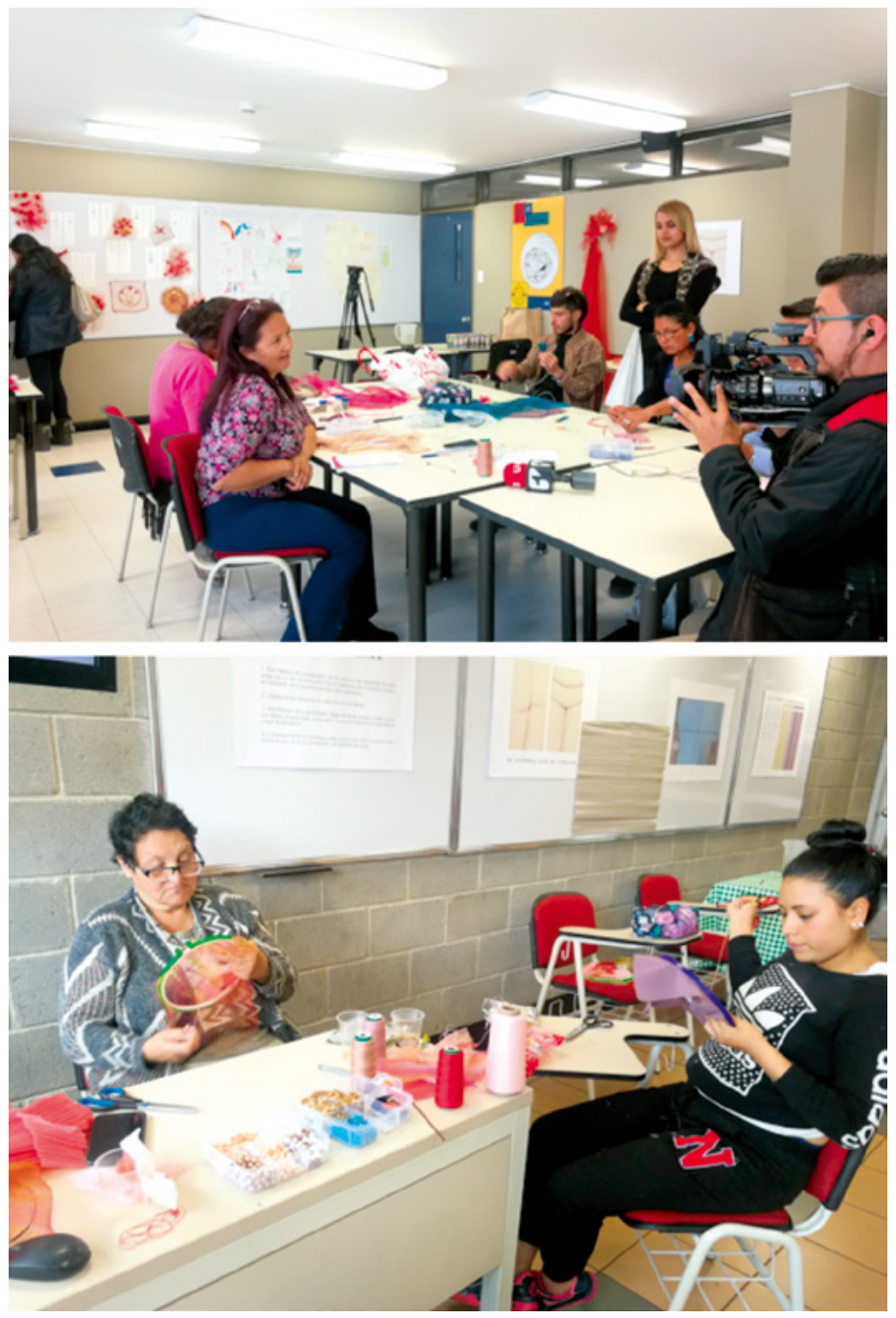

Figura 2. Taller de conceptualización-Laboratorio vivo. 
"Mi historia aún no termina" es un caso de creación denominado laboratorio vivo, en el que el taller de diseño se concibe como un espacio social de coexistencia o cohabitación de diferentes actores del conflicto para la reflexión y la creación, buscando trascender e impactar socialmente.

El concepto de laboratorio vivo fue utilizado anteriormente por German Molina (2017), quien propone reunir estratégicamente a las personas para que resuelvan conjuntamente problemas y planeen estrategias. En este caso, se sumó la metodología de diseño, como la manera de contar y liberar parte de la carga del conflicto y tratar de liberarse y resignificarse como sujeto.

Este proyecto no se enfocó exclusivamente en un desarrollo estético de prendas como resultado, sino en un proceso de reflexión donde, si bien existió el resultado, no era lo más importante. En cambio, a partir de la conceptualización de la trama social y el individuo inmerso en ella, el trabajo se centró en conceptualizar el conflicto y el yo bajo metodologías propias del diseño de modas y textil, como la generación de paneles visuales, autorrepresentaciones, construcción de códigos y lenguaje por medio de experimentaciones textiles. De esta forma, el hacer del taller se abrió a un espacio colaborativo y cooperador para la paz.

\subsection{Metodología propuesta para los talleres}

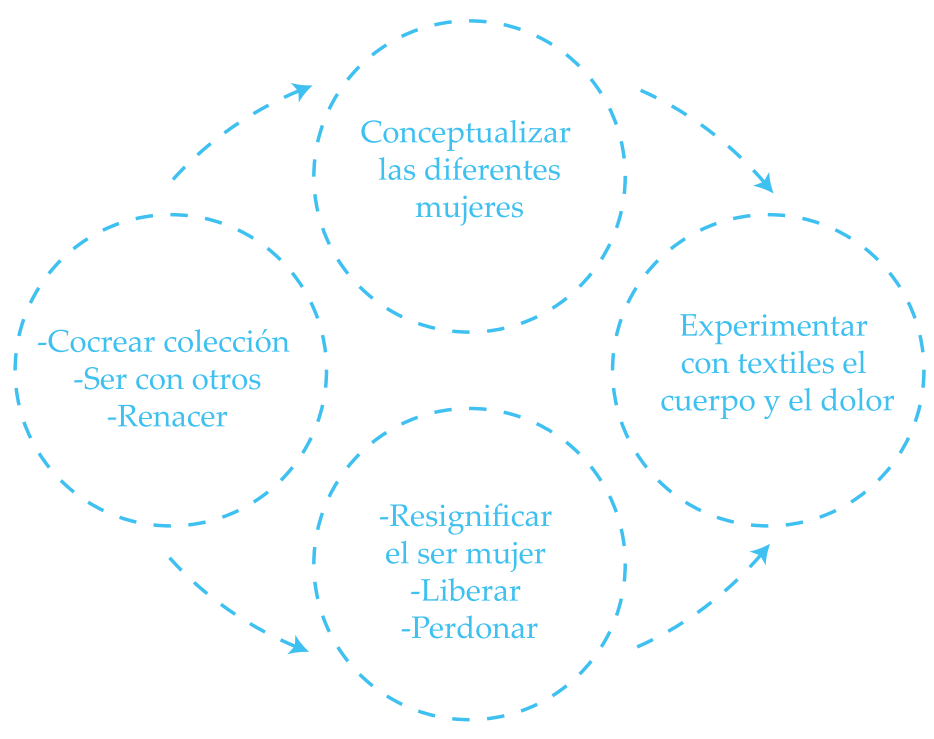

Figura 3. Metodología del taller. Fuente: autoras. 
En primera instancia, el objetivo fue desarrollar una serie de experimentaciones textiles y piezas conceptuales que narraran la reflexión y la vivencia de los cuerpos de mujer, el género y la violencia; de esta manera se generaron nudos, rasgados, rupturas, fieltrados, mezclas de colores que se bordaron y tejieron como retazos de historias de diferentes sujetos, piezas textiles convertidas en obras y sujetos como una gran historia para todos, las mujeres se expresaron con bordados experimentales, tejidos y recamados que configuraron telas abstractas y construyeron una obra que narró el caos en la vida de cada mujer en el conflicto.

\subsection{Fases de cocreación en laboratorio}

Como en la primera etapa se trabajó con una serie de talleres de reflexión y reconocimiento de su identidad y resignificación o valoración de la misma con más mujeres, espacio propicio para sentir la experiencia del ser único y representar con una mirada positiva y de perdón la historia, en esta fase de cocreación surgieron paneles creativos para la ideación, dirigidos a excombatientes y víctimas del conflicto armado. El propósito principal fue hacer una representación, por medio de experimentaciones textiles y bordados, de las experiencias individuales de cada participante desde su historia, sea con dolor, placer o incertidumbre del cuerpo, sexualidad y género, construyendo así mecanismos de liberación, respeto e igualdad $\mathrm{y}$, en consecuencia, paz.

La segunda fase fue un espacio experimental para la creación, donde se construyó una pieza artística experimental con la colaboración de todos los participantes del taller, con el fin de realizar una exposición, que sumara las experiencias individuales de excombatientes y víctimas mediante la pieza de arte colaborativa e incluyente, siendo cada una de las piezas individuales construida con diferentes materiales y técnicas, dando la oportunidad de visibilizar diversas miradas sobre la violencia de género en el conflicto armado en Colombia. Este espacio empoderó y le dio voz y representación a los cuerpos que construyeron y compartieron sus relatos en "Mi historia aún no termina". 
La tercera fase consistió en la creación de una colección de indumentaria íntima, como segunda piel; se trató de un espacio de sentir la experiencia de vivir su historia en la piel, en su cuerpo y en su intimidad, donde las excombatientes y víctimas pudieron tener diferentes conocimientos básicos para la creación de esta piezas mediante un ejercicio de patronaje sobre el cuerpo y creación sensible a partir de la propia inspiración. Así se logró hacer una reflexión sobre cuerpo, sexualidad y género desde la experiencia individual de las participantes; $\mathrm{y}$ crear conjuntamente el concepto y valores formales de la colección (forma, color, textura), con intervención de ornamentaciones por parte de las personas involucradas.

\section{La puesta en marcha de los talleres}

Los talleres fueron abordados con la idea de fomentar el empoderamiento y autoconfianza que debe crecer alrededor de los cuerpos de las excombatientes y víctimas del conflicto armado en Colombia, y con este fortalecer la identidad y género de cada sujeto, dada la importancia de reconocerse como un ser social libre que merece amor y respeto en cualquier contexto.

Asistieron alrededor de 15 personas a cada taller, los cuales estuvieron compuestos por diferentes actividades como bordar, tejer, anudar, rasgar y narrar a través del textil, permitiendo la fácil adquisición de conceptos y habilidades necesarias para el desarrollo de la cocreación. MHANT extendió estas actividades de forma dinámica y entretenida para los asistentes, pues no solo pretendía generar habilidades en el diseño y bordado, sino que se concentró en que cada cuerpo y cada experiencia hablara y expresara su naturaleza única. La buena comunicación entre los participantes del proyecto se logró gracias a la relación construida entre ellos y el buen trabajo en equipo.

Después de lograr que los participantes abrieran sus historias al grupo y encontraran la confianza para hablar de cada tema con sinceridad y honestidad, se logró el reconocimiento de los valores y los conceptos que se plasmarían de forma simbólica en piezas de corsetería y otras piezas experimentales donde ellas pudieron visibilizar y exteriorizar sus experiencias. Sumado a lo anterior, mediante diferentes técnicas que se enseñaron en los talleres, los participantes pudieron reforzar y adquirir nuevas habilidades que les permitirán entrar al campo laboral.

Además, se pretendía revivir la confianza en sí mismas y brindarles más importancia de existir como individuos irrepetibles; pero sobretodo, de eliminar prejuicios sobre el cuerpo y la sexualidad, como fuente de positivismo para enfrentar el futuro. De 
esta manera, el diseño de modas y el diseño textil han sido parte importante de la reconstrucción del tejido social, sobretodo, porque sirve de plataforma de interacción entre sujetos, de diálogo, para que cada una de las excombatientes y víctimas del conflicto haga su reflexión, e incluso una limpieza emocional por medio de la experimentación textil, basada en la introspección a través del desarrollo de una metodología experimental de creación que sanó heridas y las revitalizó como personas.

\section{Los desafíos del diseño para el desarrollo social}

El primer taller fue determinante para ganar la confianza. En el primer encuentro se dieron a conocer las cualidades de las integrantes del proyecto. Se expusieron logros y experiencias personales creando los primeros lazos del equipo. De esta forma se lograron construir bases sólidas para el desarrollo del proyecto como la confianza, la seguridad y el respeto.

El segundo reto fue acabar con la culpa, el miedo y la vergüenza a hablar de género y sexualidad con personas mayores o mujeres víctimas de violencia sexual, así como entender hechos y quitar culpas, lograr la apertura de los participantes y ganar su confianza para que compartieran sus historias con los demás miembros del grupo y sanaran la idea social de culpa y vergüenza.

Todo proyecto necesita un equipo de apoyo que se permita dejar de ser individuo para ser parte de un equipo o sistema, una esfera transformadora y creativa, como enuncia el conocido refrán chino: "Solo se llega más rápido, juntos se llega más lejos". Así, la creación motivada desde una esfera o sistema de diseño transformador parte de lograr un equipo interdisciplinario que sea capaz de trabajar sobre diferentes perspectivas para el desarrollo social, lo psicológico, lo creativo y lo técnico. Es por esto que el proyecto encontró varios contactos que fueron fundamentales para el desarrollo interdisciplinar de los talleres. 
En primera instancia, se buscó una institución educativa que fuera inclusiva y plural. La Universidad Jorge Tadeo Lozano ofreció sus instalaciones para el desarrollo de los talleres y para dar la oportunidad a las mujeres y hombres del conflicto de tener un espacio para aprender y reflexionar en la universidad como estudiantes cotidianos; fue entonces un sitio oficial para la socialización de la experiencia y una fuente de financiación para este proyecto.

De igual forma se contó con el soporte de estudiantes de Derecho de la Universidad Autónoma de Colombia y Pentagon Wave, una organización internacional que realiza una investigación transdisciplinaria para desarrollar, aplicar y entregar productos y servicios innovadores, como alternativas poderosas en salud mental y resolución de conflictos.

Además, el proyecto contó con el apoyo de las fundaciones Rosa Roja y Tejiendo Paz, que por su trayectoria trabajando en el postconflicto encontraron en el proyecto una buena iniciativa y decidieron apoyarla con los espacios para desarrollar las actividades propuestas. 


\section{Conclusiones y resultados}

El método de diseño es extrapolable como método para la resignificación y creación para el desarrollo y la transformación social, donde se puede proponer a los sujetos y sus entramados sociales como ejes conceptuales para la innovación y el desarrollo creativo de sus actores, generando incluso la posibilidad de emprendimientos creativos y tecnologías sociales de producción.

El desarrollo de proyectos de diseño bajo la perspectiva social hace del diseño una plataforma para la restauración de individuos y tejido social, el cual puede dar posibilidades para la conformación de cadenas productivas cortas, diseño de producto con identidad local y formas de producción más amigables.

El concepto de mujer, cuerpo, sexualidad y género pueden ser vistos desde una mirada feminista, fuera de miradas politizantes, normativas o conservadoras, así vistos plenamente desde la reconstrucción individual, pero que de ellos depende la posibilidad de autorrepresentación y significación para la resocialización, lo que promueve el autoestima, la aceptación y el derecho a ser.

Entender el papel del diseño y el diseñador de moda y textiles en la esfera nacional de conflicto y postconflicto como agentes de transformación social propone al campo del diseño como una disciplina y un sistema activo bajo el humanismo y el pensamiento responsable para la sociedad que contribuye a contrarrestar la precariedad y la desigualdad.

Aplicar la metodología de conceptualización de situaciones y personas para la ideación en el diseño es una manera de reflexionar sobre sí mismo o sobre contextos que, junto al mapa de actores, construye un laboratorio vivo, en este caso, víctimas y excombatientes, pero sobre todo personas. Aquí se dio espacio a la generación de ideas y posteriores piezas de arte y otros productos bajo una experimentación textil que se basó en la exteriorización o materialización del desahogo y, como se dijo anteriormente, sirvió de perdón e higiene social, proponiendo valores integradores y resocializadores en las prácticas y dinámicas creativas.

MHANT como proyecto de diseño social arrojó resultados progresivamente, pues en los primeros encuentros se establecieron lazos de confianza que permitieron que los temas de sexualidad, cuerpo y género fueran permeando los talleres poco a poco, hasta que las integrantes se sintieran cómodas compartiendo su experiencia al grupo. Se aclararon los conceptos de cuerpo, sexualidad y género, socializando los conocimientos y experiencias individuales de las asistentes. 
Al finalizar los talleres las participantes tenían mucho más conocimiento y hablaban con mayor fluidez acerca de su género, cuerpo e incluso sexualidad; así son sujetos liberados. De igual forma, el taller permitió que las participantes se expresen a su ritmo, generándoles empoderamiento y seguridad en sí mismas.

La reincorporación de excombatientes y víctimas del conflicto armado en Colombia requiere del tratamiento de todo tipo de secuelas psicológicas, para lo cual los ejercicios asociados a los talleres comenzaron por abordar el perdón, la sanación y la reflexión por medio de la búsqueda de la verdad. Comprender el contexto en el que se desarrollaron sus historias y compartir esa verdad es base fundamental para la reparación y la paz.

La reintegración de víctimas y excombatientes es un espacio de reconstrucción posbélica que necesita el compromiso de diferentes agentes sociales, pues el Estado no logra llenar todas las esferas. Por esto, la cooperación y participación de cada individuo es fundamental para lograr un proceso completo. En este sentido el proyecto "Mi historia aún no termina" permite que nuestro rol como diseñadores incluya ser agentes de cambio, para el cual nuestros talleres se convierten en un espacio de cooperación.

Para MHANT, lo anterior es una estrategia adicional para la paz que implica sortear diferentes barreras: la primera es personal, el volver a sentirse social, humano y sujeto; la segunda, superar las barreras sociales de la desconfianza, del juzgamiento y entrar a ser parte de la sociedad, en este caso como personas, como ciudadanos; y, la tercera, la inclusión social, donde el tejido debe ser hecho con la intención de reconstruir y generar espacio para sanar. 


\section{REFERENCIAS BIBLIOGRÁFICAS}

Bourdieu, P. (2002). La distinción criterio y bases sociales del gusto. Recuperado de http://biblioteca. udgvirtual.udg.mx/eureka/pudgvirtual/Bourdieu2.pdf

Butler, J. (2007). El género en disputa. (Paidos, Ed.). Recuperado de http://www.equidad.org.mx/images/ stories/documentos/genero_en_disputa.pdf

Horta, A. (2016). Investigación: un nodo teorético del diseño. August. Recuperado de https://doi.org/10.17151/ kepes.2015.12.11.6

Jiménez, C. (2016). Las mujeres en los conflictos armados: conflicto, proceso de paz y posconflicto. U. de M. Publicaciones y Divulgación científica, Ed.

Ledesma, del V. M. (2013). Cartografía del Diseño Social. Aproximaciones conceptuales, 43(1), 97-106.

Atehortúa Cruz, A. L. \& Rojas Rivera, D. M. (2008). El narcotráfico en Colombia. Pioneros y capos. Dialnet.

Molina Garrido, G; Mendoza Simmonds,L; Ortega Hoyos, A. (2017). Tecnologías simbólicas y culturales creativas. Universidad de Bogotá Jorge Tadeo Lozano: Bogotá.

Segato, R. L. (2014). Las nuevas formas de la guerra y el cuerpo de las mujeres, 29, 341-372.

Terenzi, P. (2012). Identidad y social en Hannah Arendt, 8.

Urteaga, E. (2010). La teoría de sistemas de Niklas Luhmann. Contrastes. Revista Internacional de Filosofía, XV(1136-4076), 301-317. 